\title{
EVALUACIÓN DE GRAMÍNEAS FORRAJERAS EN LA ZONA ALTA LECHERA DE COSTA RICA
}

\author{
María Mesén ${ }^{1}$ \\ William Sánchez ${ }^{1}$
}

\begin{abstract}
RESUMEN
La presente investigación se realizó en una finca ubicada en el distrito Cot, cantón Oreamuno, provincia Cartago. La topografía de la finca es irregular y la altitud es de 2.100 msnm, la temperatura y precipitación promedio anual son de $20,7^{\circ} \mathrm{C}$ y $2.121 \mathrm{~mm}$, respectivamente. La siembra se llevó a cabo el 14 de Setiembre de 1996. El objetivo fue evaluar la adaptabilidad de una colección de doce cultivares de gramíneas de los géneros, Lolium, Festuca, Phalaris y Dactylis y además el Pennisetum clandestinum como testigo. El diseño experimental utilizado, fue de bloques completos al azar con un arreglo de parcelas divididas. Se evaluaron dos cultivares anuales de pastoreo, dos perennes de corte y nueve perennes de pastoreo. Los cultivares anuales no se adaptaron a la zona ya que presentaron bajos rendimientos e invasión de plagas. Los bajos rendimientos de los cultivares de corte demuestran la poca adaptabilidad de los mismos a la zona. Los cultivares perennes de pastoreo, recibieron cortes cada seis semanas durante dos años, al último corte sobrevivieron únicamente cuatro gramíneas, el Lolium perenne, Aberolite, los Festuca arundinacea, Fawn y Manade y el testigo local (Pennisetum clandestinum) Los valores promedio de producción de biomasa de los cultivares persistentes, oscilaron entre 2,6 y 3,1 y entre 0,5 y 1,9 toneladas de materia seca por hectárea por corte durante la época lluviosa y seca respectivamente. El forraje que presentó la menor disminución en la producción de biomasa durante la época seca fue el testigo local (Pennisetum clandestinum) con una reducción del 30\%, lo cual es una característica favorable en los forrajes perennes, además mostró el mayor rendimiento de materia seca durante la época de poca precipitación. El análisis de los resultados para los valores de materia seca de los cultivares perennes que sobrevivieron, se hizo por medio de un análisis de varianza, el cual demostró que no hay deferencias significativas entre los mismos ( $P=0,6)$. Para los demás cultivares se utilizaron medidas de tendencia central. Los cultivares perennes de piso que sobrevivieron no superaron al testigo local ( Pennisetum clandestinum), por lo tanto no se recomiendan para la zona en estudio. Se debe continuar investigando con otros cultivares de uso potencial en sistemas de producción de leche de altura.
\end{abstract}

Palabras clave: Forrajes, gramíneas, adaptación, valor nutritivo, producción de leche.

\section{INTRODUCCIÓN}

La ganadería de leche es una de las principales actividades agropecuarias en Costa Rica, ya que los productos lácteos son básicos en la dieta de la población; además representa un rubro de mucha importancia en la economía nacional, logrando llenar la demanda interna y generando divisas provenientes de las exportaciones (Villegas 1993).

Sin embargo debe tenerse presente que los productores de Costa Rica tendrán que enfrentar la problemática de la competitividad que encierra la economía de escala, en comparación con los inmensos volúmenes de

\footnotetext{
${ }^{1}$ Instituto Nacional de Innovación y Transferencia en Tecnología Agropecuaria ( INTA), Costa Rica.
} 
producción e industrialización en naciones tradicionalmente exportadoras, como Uruguay, Argentina y Nueva Zelanda con niveles que oscilan entre 2,4 y 14 millones de kilogramos por planta por día, mientras que el de la planta que más procesa en Costa Rica es de 927.000 kilogramos por día (Villegas 2004).

Además la producción de leche en áreas tropicales depende en gran medida de los forrajes, principalmente gramíneas, los cuales siguen siendo la fuente alimenticia más común y de menor costo, aún cuando frecuentemente no proporcionan la cantidad y/o calidad que requieren los animales de mediana y alta producción.

En estos casos en la mayoría de las empresas, las necesidades nutricionales se satisfacen mediante el uso de alimentos balanceados, basados en materias primas importadas lo cual encarece los costos de producción en el rubro de alimentación, representando éstos hasta un $72,0 \%$ de los costos totales de alimentación en ganadería de leche especializada (MAG;IICA 1991).

Las especies de gramíneas de los géneros Lolium, Festuca, Dactylis y Phalaris son forrajes de alto rendimiento y valor nutritivo y la mayoría se adaptan a alturas superiores a los $2.000 \mathrm{msnm}$ y a temperaturas inferiores a $22^{\circ} \mathrm{C}$ (Bernal 1991). El género Lolium abarca variedades anuales (Lolium multiflorum) y perennes Lolium perenne y a partir de éstos, se pueden obtener híbridos (Lolium multiflorum) $\times$ (Lolium perenne)(Bernal 1992). Los Festuca, Dactylis y Phalaris son gramíneas perennes; los dos primeros se usan generalmente para pastoreo y el último para corte (Bernal 1991).
El Kikuyo (Pennisetum clandestinum) es el forraje de pastoreo más difundido en Costa Rica en las zonas altas dedicadas a la producción de leche. Es una gramínea herbácea, perenne, rastrera con estolones superficiales y subterráneos (Garza et al. 1973, consultado por Vargas 1981).

Por lo anterior, se pretende evaluar una colección de gramíneas de los géneros mencionados; con el Kikuyo (Pennisetum clandestinum) como testigo local, con el fin de buscar cultivares de gramíneas sobresalientes que se adapten a la zona alta lechera de Costa Rica.

\section{MATERIALES Y MÉTODOS}

El estudio se desarrolló en una finca ubicada en el distrito Cot, cantón Oreamuno, provincia de Cartago, a $83^{\circ} 53^{\prime} 30^{\prime \prime}$ longitud oeste y a $9^{\circ}$ $57^{\prime}$ latitud norte. La topografía de la finca es irregular y se encuentra ubicada a una altitud de $2.100 \mathrm{msnm}$. La temperatura y precipitación promedio anual son de $20,7^{\circ} \mathrm{C}$ y 2.121 $\mathrm{mm}$, respectivamente.

El terreno utilizado había sido cultivado anteriormente con Pennisetum clandestinun. Geomorfológicamente el suelo es de origen volcánico y taxonómicamente corresponde al orden de los andisoles (Bertsch et al. 1993).

El análisis de suelo (Cuadro 1) muestra valores adecuados de $\mathrm{pH}$ y aluminio, como consecuencia el porcentaje de acidez es 1,31. Borel (1981) menciona que los valores de acidez inferiores a 25,0 \% son adecuados para gramíneas. Los contenidos de $\mathrm{Ca}, \mathrm{Mg}, \mathrm{K}$, P, los microelementos y las relaciones se encuentran en un rango óptimo, mientras que el $\mathrm{Fe}$ se encuentra en un nivel alto (Bertsch 1987).

Cuadro 1. Resultado del análisis de suelo antes de la siembra. Cartago. 1996. ${ }^{1}$

\begin{tabular}{cccccccccccc}
\hline \multicolumn{4}{c}{ Meq/100ml suelo } & \multicolumn{1}{c}{ Ug/ml suelo } & & Textura & Materia Orgánica \\
pH & Al & Ca & Mg & K & P & Zn & Mn & $\mathbf{C u}$ & Fe & & \\
6,0 & 0,15 & 7,8 & 2,0 & 1,5 & 15,0 & 19,9 & 13,0 & 32,0 & 208,0 & Franco & 11,3 \\
\hline
\end{tabular}

${ }^{1}$ Fuente: Laboratorio de Suelos, Foliares y Aguas. INTA. 
La siembra se realizó el 14 de Setiembre de 1996 con semilla sexual de gramíneas forrajeras para clima frío (Cuadro 2). Se hizo corte de uniformización al inicio de las lluvias del año siguiente y se hicieron evaluaciones de producción de biomasa, altura, cobertura e incidencia de plagas y enfermedades cada seis semanas durante dos años.

La metodología de evaluación que se empleó fue una modificación de la utilizada por la Red Internacional de Evaluación de Pastos Tropicales (RIEPT), (Toledo 1982). Los cambios realizados a la metodología consistieron en una disminución en el tamaño de las parcelas y del marco para muestrear el cual fue de 0,5 $\mathrm{m} \times 1,0 \mathrm{~m}$ con cuadrículas de 0,25 $\mathrm{m} \times 0,25 \mathrm{~m}$. Además se usó una escala de uno a cuatro para plagas en general y no una para insectos y otra para enfermedades como señala la metodología.

Cuadro 2. Colección de gramíneas evaluadas. Cartago. 1996 -1998.

\begin{tabular}{cccc}
\hline Cultivares & Simbología & Comportamiento & Procedencia \\
Lolium hybridum, Bison & RB & Perenne de pastoreo & U.S.A. \\
Lolium multiflorum, Tama & RT & Anual de pastoreo & Chile \\
Lolium multiflorum, Grazer & RG & Anual de pastoreo & U.S.A. \\
Lolium perenne, Aberolán & RA & Perenne de pastoreo & Inglaterra \\
Lolium perenne, Nui & RN & Perenne de pastoreo & Chile \\
Lolium perenne, Aberolite & RI & Perenne de pastoreo & Inglaterra \\
Festuca arundinacea, Fawn & FF & Perenne de pastoreo & Chile \\
Festuca arundinacea, Manade & FM & Perenne de pastoreo & Chile \\
Phalaris aquatica, Sirolán & PS & Perenne de corte & Chile \\
Phalaris aquatica, Sirosa & PI & Perenne de corte & Chile \\
Dactylis glomerata, Currie & OC & Perenne de pastoreo & Chile \\
Dactylis glomerata, Potomac & OP & Perenne de pastoreo & Chile \\
Testigo (Pennisetum clandestinum) & TL & Perenne de pastoreo & Costa Rica \\
\hline
\end{tabular}

Descripción de la unidad experimental

La parcela experimental fue de $6,0 \mathrm{~m}^{2}$ con tres hileras de 3,0 $\mathrm{m}$ de largo y distanciadas entre ellas a 0,5 m. Los muestreos se hicieron en la hilera central, dejando los otros surcos como efecto de borde.

\section{Fertilización}

Las dosis empleadas fueron $100 \mathrm{~kg} / \mathrm{ha}$ de $\mathrm{N}, 50 \mathrm{~kg} / \mathrm{ha}$ de $\mathrm{P}_{2} \mathrm{O}_{5}, 50 \mathrm{~kg} / \mathrm{ha}$ de $\mathrm{K}_{2} \mathrm{O}, 20 \mathrm{~kg} / \mathrm{ha}$ de $\mathrm{Mg}$ y $20 \mathrm{~kg} / \mathrm{ha}$ de $\mathrm{S}$. Los fertilizantes se aplicaron como lo indica la metodología de la siguiente manera: el fósforo, magnesio y azufre se incorporaron al suelo a la siembra, el nitrógeno y el potasio se aplicaron fraccionados, con 1/3 de lo recomendado cuatro semanas después de la siembra, $1 / 3$ después del corte de uniformización y el último tercio al cabo de dos cortes (Toledo, Schultze-Kraft 1982). 


\section{Variables evaluadas}

\section{Producción de biomasa y calidad nutritiva}

Las evaluaciones se realizaron cada seis semanas después del corte de nivelación, la altura de corte fue de aproximadamente 5,0 $\mathrm{cm}$ sobre el nivel del suelo. Los cortes se efectuaron a razón de $1 \mathrm{~m}$ en la hilera central de cada parcela, dejando sin cortar los extremos, como efecto de borde (Roig 1989).

El material de la hilera central se pesó en verde y luego se obtuvo una sub-muestra de 500 gramos para la determinación del contenido de materia seca (M.S.). Se utilizó una muestra compuesta de las tres repeticiones para la determinación de proteína cruda (P.C.) y digestibilidad in vitro de la materia seca (D.I.V.M.S.). Debido a esta situación se hizo análisis de varianza solamente para la producción de biomasa.

\section{Cobertura}

Esta variable se evaluó como porcentaje del área que no presentó suelo desnudo. Las evaluaciones coincidieron con los muestreos de rendimiento. Para este propósito, se empleó la metodología propuesta por la RIEPT (Toledo y Schultze-Kraft 1982), con la diferencia de que al tener las parcelas sólo tres hileras, de las cuales una constituye la parcela útil, se usó un marco rectangular de $0,5 \mathrm{~m} \times 1,0 \mathrm{~m}$ con cuadrículas de 0,25 m x 0,25 m.

\section{Altura}

Para esta medición, se tomó la altura desde el nivel del suelo hasta el punto más alto de la planta, sin estirarla y sin considerar la inflorescencia (Toledo, Schultze-Kraft 1982).

Plagas (Insectos y enfermedades) (Adaptado de Calderón 1982 y Lenne 1982)

Se utilizó una escala visual de uno a cuatro en la cual:

1. Presencia ( $5 \%$ del follaje afectado).

2. Daño leve (5\%-20\% del follaje afectado).

3. Daño moderado $(20 \%-40 \%$ del follaje afectado).

4. Daño grave (más de $40 \%$ del follaje afectado).

\section{RESULTADOS Y DISCUSIÓN}

\section{Producción de biomasa y valor nutritivo}

\section{Gramíneas anuales}

Los cultivares anuales de pastoreo (Cuadro 3) fueron cosechados al inicio de la floración, (194 y 109 días para Lolium multiflorum, Tama y Grazer respectivamente) el comportamiento fue similar entre ellos, el valor nutritivo, la altura y la cobertura fueron altos, sin embargo los rendimientos de materia seca fueron muy parecidos a los de los forrajes perennes en estudio (Cuadro 5) los cuales no requieren ser sembrados anualmente. Por lo anteriormente mencionado es preferible utilizar los forrajes perennes ya que el costo del forraje producido es inferior.

Cuadro 3. Comportamiento de las gramíneas anuales al inicio de la floración. Cartago. 1997.

\begin{tabular}{lcc}
\hline Información obtenida & \multicolumn{2}{c}{ Cultivar } \\
& RT & RG \\
Inicio de floración, & 194 & 109 \\
días crecimiento & & \\
Proteína cruda, \% & 17,0 & 17,1 \\
Digestibilidad in vitro & 79,0 & 80,3 \\
de la materia seca, \% & & \\
Materia seca, t/ha & 2,5 & 2,4 \\
Altura, cm & 50 & 50 \\
Cobertura, \% & 100 & 100 \\
Follaje afectado (Puccinia sp),\% & $20-40$ & $20-40$ \\
Follaje afectado (Collarea oleosa), \% & 5 & 5 \\
\hline
\end{tabular}

\section{Gramíneas de corte}

Los cultivares de corte presentaron rendimientos promedio anuales de materia seca de 3,68 y 4,22 toneladas por hectárea por corte, lo cual significa una disminución de 60 y $78 \%$ durante la época seca para Phalaris aquatica, Sirolán y Sirosa respectivamente, (Cuadro 4). En general los rendimientos de materia seca reportados, son inferiores a los de otros forrajes de corte existentes en la zona como por ejemplo el King grass (Pennisetum purpureun $\mathrm{x}$ Pennisetum typhoides) del cual se reportan rendimientos 
de materia seca de hasta 20,0 toneladas por hectárea por corte (MAG;IICA 1991). En cuanto al valor nutritivo de estos forrajes, se puede observar en el mismo Cuadro que, la proteína cruda y la DIVMS son altos, sin embargo al ser bajos los rendimientos de materia seca, el aporte total de proteína cruda y de materia seca digestible por hectárea también son bajos.

Cuadro 4. Valores promedio del rendimiento y valor nutritivo de las gramíneas de corte. Cartago. 1998.

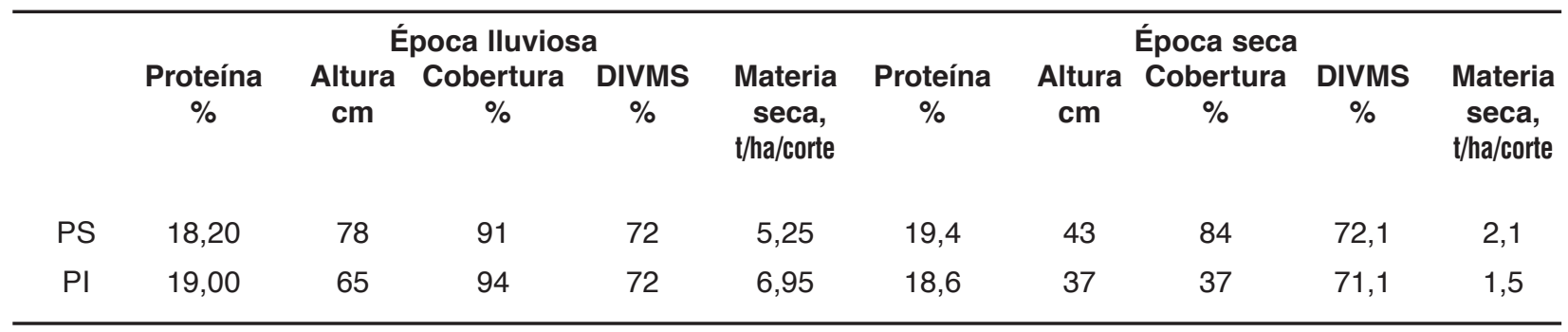

\section{Gramíneas perennes de pastoreo}

Los valores promedio de producción de biomasa de los cultivares persistentes, oscilaron entre 2,6 y 3,1 y entre 0,5 y 1,9 toneladas de materia seca por hectárea por corte durante la época lluviosa y seca respectivamente. El forraje que obtuvo la menor disminución en la producción de biomasa durante la época seca fue el testigo local (Pennisetum clandestinum) con una reducción del $30 \%$ lo cual es una característica favorable en los forrajes perennes. Además presentó el mayor rendimiento de materia seca durante la época de poca precipitación (Cuadro 5).

El análisis de varianza para las determinaciones mencionadas, mostró que no hay diferencias entre los cultivares que sobrevivieron durante todo el período de muestreo $(P=0,6)$. En cuanto al contenido de proteína cruda (Cuadro 5) se analizaron muestras compuestas de las tres repeticiones, utilizando medidas de tendencia central y se obtuvieron valores muy similares entre los diferentes cultivares. Los rendimientos de materia seca de los cultivares en estudio fueron semejantes a los reportados por Sánchez y Mesén, 2004 y Mesén y Sánchez, 2004.

Cuadro 5. Valores promedio del rendimiento y valor nutritivo de las gramíneas perennes durante el período de corte. Cartago. 1998.

\begin{tabular}{|c|c|c|c|c|c|c|c|c|}
\hline \multirow[b]{2}{*}{ Cultivar } & \multicolumn{4}{|c|}{ Época lluviosa } & \multicolumn{4}{|c|}{ Época seca } \\
\hline & Proteína,\% & $\begin{array}{l}\text { Altura, } \\
\text { cm }\end{array}$ & $\begin{array}{c}\text { Cobertura, } \\
\%\end{array}$ & $\begin{array}{c}\text { Materia } \\
\text { seca, } \\
\text { t/ha/corte }\end{array}$ & $\begin{array}{c}\text { Proteína, } \\
\%\end{array}$ & $\begin{array}{l}\text { Altura, } \\
\text { cm }\end{array}$ & $\begin{array}{c}\text { Cobertura, } \\
\%\end{array}$ & $\begin{array}{l}\text { Materia } \\
\text { seca, } \\
\text { t/ha/corte }\end{array}$ \\
\hline $\mathrm{RB}$ & $P$ & & & & & & & \\
\hline RA & $P$ & & & & & & & \\
\hline $\mathrm{RI}$ & 17,9 & 40,5 & 95,0 & 2,9 & 18,2 & 30,0 & 90,0 & 1,5 \\
\hline RN & $P$ & & & & & & & \\
\hline $\mathrm{FF}$ & 19,1 & 37,0 & 89,0 & 3,1 & 19,1 & 25,0 & 80,0 & 1,6 \\
\hline FM & 18,1 & 39,0 & 85,0 & 2,6 & 18,1 & 20,0 & 75,0 & 0,5 \\
\hline OC & $\mathrm{P}$ & & & & & & & \\
\hline OP & $P$ & & & & & & & \\
\hline TL & 18,8 & 39,0 & 95,0 & 2,7 & 17,4 & 30,0 & 90,0 & 1,9 \\
\hline
\end{tabular}

$\mathrm{P}=$ perdido. 


\section{Cobertura y Altura}

\section{Gramíneas anuales de pastoreo}

El porcentaje de cobertura fue alto para ambos cultivares, en los cuales se presentó un $100 \%$ del suelo cubierto. La altura de las plantas fue de $50 \mathrm{~cm}$ también para los dos cultivares anuales evaluados (Cuadro 3 ).

\section{Gramíneas perennes de corte}

Durante la época lluviosa los valores de altura fueron 78 y $65 \mathrm{~cm}$ y los de cobertura 91 y 94 \% para Phalaris aquática, Sirolán y Sirosa respectivamente, sin embargo, durante la época de mínima precipitación hubo una disminución drástica en ambos forrajes en cuanto a altura. En lo que a cobertura se refiere, la mayor disminución se dio en Phalaris aquatica, Sirosa con una reducción del 60\% (Cuadro 4).

\section{Cultivares perennes de pastoreo}

Los valores de cobertura y altura durante la época lluviosa fueron muy similares y oscilaron entre 85,0 y $95,0 \%$ y entre 37,0 a $40,5 \mathrm{~cm}$ respectivamente. Durante la época seca hubo una reducción de todos los valores de altura, el que menos disminuyó fue el testigo local (Pennisetum clandestinum) con un descenso del $23 \%$. En cuanto a la cobertura en general la reducción fue baja, el que disminuyó más fue el Festuca arundinacea, Manade con un 12\% (Cuadro 5).

\section{Plagas}

Los cultivares anuales de pastoreo fueron afectados por plagas, el daño ocasionado por Puccinia sp fue moderado o sea aproximadamente un $40 \%$ del follaje fue dañado, dicho porcentaje podría variar de acuerdo con las condiciones ambientales (Cuadro 3). También hubo presencia de Collarea oleosa (5\% del follaje con lesiones).

En los cultivares perennes de pastoreo que persistieron y en los cultivares de corte hubo presencia ( $5 \%$ del follaje afectado) de Collarea oleosa y Puccinia $s p$.

\section{CONCLUSIONES Y RECOMENDACIONES}

Los cultivares anuales no se adaptaron a la zona ya que presentaron bajos rendimientos e invasión de plagas.

Los bajos rendimientos de los cultivares de corte demuestraron la poca adaptabilidad de los mismos a la zona.

Las gramíneas que subsistieron durante todo el período de evaluación fueron el Lolium perenne aberolite y los Festuca arundinacea Fawn y Manade con rendimientos de materia seca y calidad nutritiva muy similares al testigo (Pennisetum clandestinum), por lo tanto no se consideran cultivares promisorios para la zona.

Se debe continuar investigando con otros cultivares de uso potencial en sistemas de producción de leche de altura.

\section{AGRADECIMIENTO}

Los autores expresan su agradecimiento al Doctor Danilo Pezo Q., y a los Ingenieros Luis Villegas Z. y Beatriz Molina B. por la orientación científica que le brindaron a este trabajo.

\section{LITERATURA CITADA}

Bernal, J. 1991. Pastos y forrajes tropicales. Editorial Banco ganadero. 2da Edición. Colombia. p. 491-524.

1992. Pastos y forrajes para Colombia. Editorial Banco Ganadero. 3ra. Edición. Colombia. p. 95-101.

Bertsch, F. et. al., 1993. Características de los principales órdenes de suelos presentes en Costa Rica. Congreso Nacional Agropecuario y de Recursos Naturales. U.C.R. Costa Rica. $78 \mathrm{p}$. 
1987. Manual para interpretar la fertilidad de los suelos de Costa Rica. Universidad de Costa Rica. 82 p.

Borel, R. 1981. Uso de los fertilizantes en pasturas. In: Producción y utilización de forrajes en el trópico. CATIE. Costa Rica. p.58-69.

Calderón, M. 1982. Evaluación del daño causado por insectos. In: Toledo, J.M. Manual para la evaluación agronómica. Red Internacional de Evaluación de Pastos Tropicales. (RIEPT), CIAT, Cali, Colombia. p. 45-56.

MAG (Ministerio de agricultura y Ganadería, CR); IICA (Instituto Interamericano de Cooperación para la Agricultura, CR).1991. Estudio a nivel nacional para mejorar la tecnología de alimentación del ganado lechero. Costa Rica. p.265.

Lenne, J. 1982. Evaluación de enfermedades en pastos tropicales. In:Toledo, J.M. Manual para la evaluación agronómica. Red Internacional de Evaluación de Pastos Tropicales. (RIEPT), CIAT, Cali, Colombia. p. 57-72.

Mesén M. y Sánchez W. 2004. Evaluación de gramíneas de los géneros Lolium y Festuca en la zona alta lechera de Costa Rica. Alcances Tecnológicos. INTA. San José. Costa Rica. Año 2. № 1. p. 7-12.

Roig, C.A. 1989. Evaluación preliminar de 200 accesiones de leguminosas forrajeras tropicales en el ecosistema de Bosque Tropical Lluvioso en Costa Rica. Guápiles, Costa Rica. Tesis Mag. Sc. CATIE, Turrialba, Costa Rica. 179 p.

Mesén M. y Sánchez W. 2004. Evaluación de gramíneas de piso de clima frío en Oreamuno de Cartago. Alcances Tecnológicos. INTA. San José. Costa Rica. Año 2 Nº1 p. 1-7.

Toledo, J.M. y Schultze - Kraft, R. 1982. Metodología para la evaluación agronómica de pastos tropicales. In: Toledo, J.M. Manual para la Evaluación Agronómica. Red Internacional de Evaluación de Pastos Tropicales (RIEPT), CIAT, Calí, Colombia. p. 91-109.

Vargas, M. 1981. Efecto de la fertilización nitrogenada sobre la productividad, contenido de proteína cruda y mineral de pasto Kikuyo (Pennisetum clandestinum) bajo pastoreo. Tesis Agronomía, UCR. Costa Rica.sp.
Villegas, L. 1993. Situación actual de la actividad lechera en Costa Rica. In: Memoria del Congreso Agronómica Nacional. Costa Rica. p.53.

-------. 2004. Factores determinantes de la productividad de fincas lecheras en pastoreo. In: Memoria del seminario de ganadería de leche. INTA. Departamento de Transferencia. Costa Rica. sp. 
ALCANCES TECNOLÓGICOS, AÑO 4, NÚMERO 1 